\title{
Topology optimization for the conduction cooling of a heat-generating volume with orthotropic material.
}

\author{
L.G. Page ${ }^{\mathrm{a}, *}$, J. Dirker ${ }^{\mathrm{a}}$, J.P. Meyer ${ }^{\mathrm{a}}$ \\ ${ }^{a}$ Department of Mechanical and Aeronautical Engineering, University of Pretoria, \\ Pretoria, Private Bag X20, Hatfield, 0028, South Africa
}

\begin{abstract}
In this paper the two dimensional numerical topology optimization of a high conductive conduit material, distributed within a heat-generating material, is investigated with regards to the effect of orthotropic materials. Specifically, materials with orthotropic thermal conductivities (different primary and secondary principal thermal conductivities).

Two cases are considered in this study, namely the optimal distribution of an isotropic conduit material within an orthotropic heat generating material; and the optimal distribution of an orthotropic conduit material within an isotropic heat-generating material. A finite volume method (FVM) code, coupled with the method of moving asymptotes (MMA); the solid isotropic with material penalization (SIMP) scheme; and the discrete adjoint method, was used to find the optimal distribution of the high conductive conduit material within the heat generating material.

For the optimal distribution of an isotropic conduit material within an orthotropic heat-generating material is was found that a heat-generating material angle $10^{\circ} \leq \theta_{0} \leq 60^{\circ}$ is preferred, for a higher thermal performance, and

\footnotetext{
${ }^{*}$ Corresponding author

Email addresses: Logan.Page@up.ac.za (L.G. Page), Jaco.Dirker@up.ac.za (J. Dirker )
} 
a heat-generating material angle $\theta_{0}<10^{\circ}$ and $\theta_{0}>60^{\circ}$ should be avoided.

For the optimal distribution of an orthotropic conduit material within an isotropic heat-generating material is was found that an optimal conduit material angle exists giving the best thermal performance (lowest $\tau_{\max }$ ). It was found that the optimal conduit material angle remains the same for different conductivity ratios and different heat-generating material angles. It was also found that the optimal conduit material angle directly corresponds to the domain aspect ratio, $\theta_{1, \mathrm{opt}}=\tan ^{-1}(2 H / L)$, with a minimum improvement of $3 \%$ and a maximum improvement of $50 \%$ of the thermal performance when using an orthotropic conduit material over that of an isotropic conduit material. A $50 \%$ improvement of the thermal performance effectively translates to either double the allowable heat generation or half the peak operating temperature of the isotropic heat-generating material.

Keywords: Topology optimization, Conduction cooling, Heat-generating volume, Orthotropic material, Two dimensional

\section{Introduction}

The strive for higher power densities in electronics has been the driving force behind many of the miniaturization efforts, augmentations and unconventional ways of extracting heat from heat-generating volumes. Of interest, in many such investigations, is the optimization of conductive cooling paths (formed by a high conductive conduit material) within a heat-generating material with the condition that only a limited amount of volume be used as the conduit material. As described by Bejan [7], this volume-to-point or volume-to-surface type problem is one whereby heat is generated volumetrically (in a low conductive volume of given size) which must be channeled 


\begin{tabular}{|c|c|c|c|}
\hline \multicolumn{4}{|c|}{ Nomenclature } \\
\hline $\mathcal{A}$ & coefficient matrix & Greek & symbols \\
\hline $\mathcal{B}$ & source term matrix & $\beta$ & material orientation angle \\
\hline$C$ & length of isothermal boundary & $\theta$ & material angle \\
\hline$f(\phi)$ & objective function & $\lambda$ & adjoint vector \\
\hline$g_{1}(\phi)$ & inequality constraint & $\tau_{\max }$ & non-dimensional thermal performanc \\
\hline$H$ & domain height & $\phi$ & design variable \\
\hline$k_{r}$ & thermal conductivity ratio & $\phi_{\max }$ & volume ratio limit \\
\hline k & thermal conductivity vector & $\Phi$ & arbitrary field \\
\hline$k_{1}, k_{2}$ & conductivity components & $\Omega$ & material domain \\
\hline$k_{x}, k_{y}$ & conductivity components & & \\
\hline$L$ & domain length & Subsc & ripts \\
\hline$N$ & increments for the penalization factor & 0 & heat-generating material \\
\hline$\dot{q}$ & heat generation rate & 1 & conduit material \\
\hline$R(\Phi)$ & residual & opt & optimum \\
\hline$s, s_{0}$ & MMA asymptote factors & & \\
\hline$T$ & temperature & Super & scripts \\
\hline$T_{0}$ & boundary temperature & $I$ & optimization iteration \\
\hline$T_{\text {ave }}$ & average temperature & $P$ & penalization factor \\
\hline$T_{\max }$ & maximum temperature & $T$ & matrix transpose \\
\hline$V$ & volume & & \\
\hline$V_{t}$ & total domain volume & & \\
\hline$x, y$ & Cartesian coordinates & & \\
\hline
\end{tabular}


(through a high conductive conduit material) either to a point or surface on the boundary of the volume.

The volume-to-point or volume-to-surface type problem thus requires finding the optimal distribution of a limited amount of conduit material to augment the transport of heat within a heat-generating volume to predefined boundary regions. An optimal conduit material architecture leads to either a reduction in peak operating temperature at a given heat generation rate; or an increase in allowable heat generation rate and heat flux for a given peak operating temperature. This, in general, leads to the overall effect of higher effective power densities within the volume.

The augmentation of the volume-to-point or volume-to-surface type problem has been approached by a number of different methods, such as: utilizing constructal theory for various geometrically shaped volumes $[1,4,7,8$, $13,27,31]$; and embedding predefined conduit material geometries within a heat-generating volume [14-16]. The optimization of predefined non-complex conduit material geometries are effective in augmenting the power densities with the added benefit of tailoring the conduit material geometry for ease of manufacturability. Such predefined geometries, however, impose restrictions on the conduit material architecture and may be far from the optimum solution as shown by Boichot et al. [10] and Song and Guo [32].

Approaches which involve shape and topology optimization have also been utilized to derive conduit material architectures. Li et al. [24, 25] investigated two-dimensional heat conduction using an evolutionary structural optimization method. Gao et al. [18] investigated two-dimensional conduction problems using a modified bi-directional evolutionary structural optimization scheme. Boichot et al. [10] investigated cellular automaton with the goal of effectively cooling a heat-generating surface by arranging the configuration 
of high conductive material links. Cheng et al. [12] and Song and Guo [32] implemented bionic optimization in the construction of highly effective heat conduction paths. $\mathrm{Xu}$ et al. [35] investigated the volume-to-point problem using simulated annealing, which proved to perform better than constructal theory and bionic optimization.

Dirker and Meyer [17], Gersborg-Hansen et al. [19], Marck et al. [26] and Zhang and Liu [36] investigated the two dimensional topology optimization of the conduit material within a heat-generating volume for isotropic material. Burger et al. [11] extended this approach to a three dimensional study for a cubic domain with an isothermal boundary. It was found that the optimal topologies, obtained for the two- and three-dimensional studies, were similar to those of natural trees. Alexandersen et al. [2, 3] also considered the topology optimization of two- and three-dimensional heat sinks cooled by natural convection, also leading to optimal topologies similar to natural trees.

In this paper, the method of moving asymptotes (MMA) [34] gradient based optimization algorithm is utilized, along with the solid isotropic with material penalization (SIMP) method and discrete adjoint method [20, 33], in order to determine the optimal conduit material architectures. Specifically, for materials with orthotropic thermal conductivities (different primary and secondary principal thermal conductivities).

\section{Numerical Model}

Figure 1 shows the two dimensional computational domain and boundary conditions for the topology optimization problem considered in this paper. The internal volume $\left(\Omega_{0}\right.$ domain), of length $L[m]$ and height $H[m]$, generates heat at a rate of $\dot{q}_{0}\left[\mathrm{~W} / \mathrm{m}^{3}\right]$ and has an orthotropic thermal conductivity of 
$\mathbf{k}_{\mathbf{0}}=\left(k_{x_{0}}, k_{y_{0}}\right)[W / m K]$. A portion of the lower boundary (length $C[m]$ ) is isothermal with a temperature of $T_{0}[K]$, while all other boundaries are adiabatic.

In order to augment the thermal performance of the system a conduit material ( $\Omega_{1}$ domain) is introduced with a high thermal conductivity of $\mathbf{k}_{\mathbf{1}}=\left(k_{x_{1}}, k_{y_{1}}\right)>\mathbf{k}_{\mathbf{0}}[W / m K]$. The conduit material $\left(\Omega_{1}\right.$ domain $)$ does not contribute towards the internal heat generation and thus only serves to conduct heat out of the system to the isothermal boundary. The volume ratio of the $\Omega_{1}$ domain to the total volume $V_{t}$ is however restricted, thus limiting the amount of conduit material:

$$
\frac{1}{V_{t}} \int_{\Omega_{1}} \mathrm{~d} V \leq \phi_{\max }
$$

where $\phi_{\max }$ is the volume ratio limit and $V_{t}=\int_{\Omega_{0}} \mathrm{dV}+\int_{\Omega_{1}} \mathrm{dV}$ is the total volume of the system.

The system is assumed to be two dimensional and steady and the governing steady-state energy equation (in vector form) is given as:

$$
\mathbf{k} \nabla^{2} T=\dot{q}
$$

where $\mathbf{k}=\left(k_{x}, k_{y}\right)$ is the orthotropic thermal conductivity (i.e. different $x$ and $y$ effective thermal conductivities) and $\nabla^{2}=\partial^{2} / \partial x^{2}+\partial^{2} / \partial y^{2}$. It is important to note that the internal heat-generation $\dot{q}$ and the thermal conductivity $\mathbf{k}$ may be spatially non-uniform and thus dependent on the system of coordinates $(x, y)$, this is discussed further in section 3 . The thermal conductivity $\mathbf{k}$ is assumed independent of temperature and $\mathbf{k}=\mathbf{k}_{\mathbf{0}}$ for the heat-generating material (the $\Omega_{0}$ domain) and $\mathbf{k}=\mathbf{k}_{\mathbf{1}}$ for the conduit material (the $\Omega_{1}$ domain).

The thermal conductivity ratio of the conduit material conductivity $\mathbf{k}_{\mathbf{1}}$ 
to the heat-generating material conductivity $\mathbf{k}_{\mathbf{0}}$ is defined as:

$$
k_{r}=\frac{\left|\mathbf{k}_{\mathbf{1}}\right|}{\left|\mathbf{k}_{\mathbf{0}}\right|}
$$

where $|\cdot|$ is the Euclidean norm.

Figure 2 shows the material orientation with respects to the system of coordinates $(x, y)$ defined in Fig. 1, where $|\mathbf{k}|$ is the effective thermal conductivity norm at a material angle $\theta$ and $\beta$ is the material orientation angle. The material, for example, may be a composite material, layered sub-materials or a crystalline sub-structure, each with different effective thermal conductivities in the materials primary and secondary principal directions (denoted by the system of coordinates $(1,2)$ defined in Fig. 2).

The effective conductivity components $k_{x}, k_{y}, k_{1}$ and $k_{2}$ can be calculated as:

$$
\begin{aligned}
& k_{x}=|\mathbf{k}| \cos (\theta) \\
& k_{y}=|\mathbf{k}| \sin (\theta) \\
& k_{1}=|\mathbf{k}| \cos (\theta-\beta) \\
& k_{2}=|\mathbf{k}| \sin (\theta-\beta)
\end{aligned}
$$

The matrial orientation angle $\beta$ is included for completeness to indicate the relationship between the materials system of coordinates $(1,2)$ and the system of coordinates $(x, y)$ used in this study. In this study it is assumed that the materials primary and secondary directions $(1,2)$ are aligned with the system of coordinates $(x, y)$, i.e. $\beta=0, k_{x}=k_{1}$ and $k_{y}=k_{2}$ and the effective thermal conductivity $\mathbf{k}=\left(k_{x}, k_{y}\right)$ will be reported as a norm $|\mathbf{k}|$ and a material angle $\theta$. 


\section{Numerical Methods}

A design variable field $\phi$ along with a variation of the solid isotropic with material penalization (SIMP) method was implemented in order to represent the heat generation $\dot{q}$ for the $\Omega_{0}$ and $\Omega_{1}$ domains:

$$
\dot{q}=\dot{q}_{0}\left(1-\phi^{P}\right)
$$

where $\phi=0$ corresponds to the heat-generating material $\dot{q}=\dot{q}_{0}$ (the $\Omega_{0}$ domain); $\phi=1$ corresponds to the conduit material $\dot{q}=0$ (the $\Omega_{1}$ domain); and $0<\phi<1$ corresponds to a composite material having both heatgenerating and conductive cooling functions. The penalization factor $P$ is introduced in order to encourage the optimization algorithm to favour design variables of either $\phi=0$ or $\phi=1$ and reduce the amount of composite material.

Similarly, the thermal conductivity $\mathbf{k}$ for the $\Omega_{0}$ and $\Omega_{1}$ domains is given as:

$$
\mathbf{k}=\phi^{P}\left(\mathbf{k}_{\mathbf{1}}-\mathbf{k}_{\mathbf{0}}\right)+\mathbf{k}_{\mathbf{0}}
$$

There are two methods for controlling this penalization factor, namely keeping the penalization factor constant throughout the MMA optimization routine or gradually increasing the penalization factor from a starting value of $P_{\text {start }}$ to a final value of $P_{\text {end }}$ over the first $N$ iterations of the optimization routine [9].

The purpose of the topology optimization is to find the optimal material distribution of the conduit material (shape of $\Omega_{1}$ ) in order to achieve the best possible thermal performance, subject to one or more constraints. The average temperature of the system was used as the objective function to 
describe the thermal performance, given as:

$$
T_{\text {ave }}=\frac{1}{V_{t}} \int_{\Omega_{0}+\Omega_{1}} T \mathrm{~d} V
$$

Other functions may also be used to describe the thermal performance of the system, for example the peak temperature $T_{\max }$ or the cost function proposed by Gersborg-Hansen et al. [19], however in the previous work by Dirker and Meyer [17] it was shown that using the average temperature as the objective function was also suitable for reducing the peak temperature. The multiobjective cost function investigated by Marck et al. [26] may also be used to find an acceptable trade-off between the variance and average temperatures.

The constrained optimization problem can formally be written as:

$$
\begin{aligned}
& \underset{w . r . t . \phi}{\operatorname{minimise}} \\
& \qquad f(\phi)=T_{\text {ave }}, \quad 0 \leq \phi \leq 1
\end{aligned}
$$

such that:

$$
g_{1}(\phi)=\frac{1}{V_{t}} \int_{\Omega_{0}+\Omega_{1}} \phi \mathrm{d} V \leq \phi_{\max }
$$

Since $\phi=0$ corresponds to the heat-generating material and $\phi=1$ corresponds to the conduit material, the volume ratio given in Eq 1 can be formally written in terms of $\phi$ to give the inequality constraint $g_{1}(\phi)$ in $\mathrm{Eq} 8$.

Equation 8 was solved using the MMA (method of moving asymptotes) optimization algorithm by Svanberg [34] with the asymptote factors $s_{0}=0.1$ and $s=0.7$. The 'dual objective function' (as described in the MMA) was solved using the Scipy [21] fmin_slsqp algorithm based on the sequential least squares programming algorithm implemented by Kraft [22]. The reader is referred to the original texts for further information. 
The gradient information of the objective function (required by the MMA algorithm) was computed using the discrete adjoint method [20, 33]:

$$
\frac{d}{d \phi} f(\phi)=-\lambda^{T} \cdot\left(\frac{\partial \mathcal{A}}{\partial \phi} \cdot T-\frac{\partial \mathcal{B}}{\partial \phi}\right)
$$

where $\mathcal{A}$ is the coefficient matrix and $\mathcal{B}$ is the source term array from the finite volume method (FVM) with the discretization of Eq. $2($ i.e. $\mathcal{A} T=\mathcal{B})$. The adjoint vector $\lambda$ was computed from:

$$
\mathcal{A}^{T} \lambda=\left[\frac{\partial}{\partial T} f(\phi)\right]^{T}
$$

where $\mathcal{A}^{T}=\mathcal{A}$ for a symmetric matrix and $\partial f(\phi) / \partial T$ is presumed specified analytically.

The governing energy equation (Eq. 2) and the adjoint equation (Eq. 9) was solved using an in-house FVM (finite volume method) code written in Python [30] and Cython [6]. This code utilized structured hexahedron elements; a harmonic interpolation scheme; and an un-corrected surface normal gradient scheme. The adjoint vector $\lambda$ (Eq. 10) and the discretized form of the governing energy equation (Eq. 2) was solved using the Scipy [21] bicgstab algorithm based on the bi-conjugate gradient stabilized iterative method with preconditioning [5].

The temperature and adjoint vector fields were initialized to zero and the design variable field were initialized to $\phi_{\max }$ (i,e. $T=0, \lambda=0$, and $\left.\phi=\phi_{\max }\right)$. The convergence criteria used for solving the temperature and adjoint vector fields was:

$$
R(T) \leq 1 e-9, \quad R(\lambda) \leq 1 e-9
$$

where $R(\Phi)=\mathcal{A} \Phi-\mathcal{B}$ is the residual for an arbitrary field $\Phi$. The convergence criteria used to terminate the optimization algorithm was:

$$
\left|f(\phi)^{I}-f(\phi)^{I-1}\right| \leq 1 e-8
$$


where $I$ is the optimization iteration.

An increasing penalization scheme, with $P_{\text {start }}=1 ; P_{\text {end }}=3$; and $N=$ 40 incremental steps was used in this paper. $P_{\text {end }}$ was selected based on the suggestions by Bendsoe and Sigmund [9] and the work by Dirker and Meyer [17]. $N$ was selected based on observation, where it was seen that at around 35 iterations the topology had become well established. The overall computational routine can be summarized and explained as follows:

Step 1: Initialize $T=0, \lambda=0, \phi=\phi_{\max }, P=1$, and $I=0$

Step 2: Compute the heat generation $\dot{q}$ and conductivity $\mathbf{k}$ from Eq. 5 and Eq. 6 respectively

Step 3: Compute $T$ from the governing energy equation (Eq. 2)

Step 4: Compute the adjoint vector $\lambda$ and gradient information $d f(\phi) / d \phi$ from Eq. 10 and Eq. 9 respectively.

Step 5: Compute the next design variable $\phi^{I+1}$ from the MMA algorithm (as described by Svanberg [34]). The MMA algorithm requires and makes use of:

- the computated average temperature $f(\phi)=T_{\text {ave }}$ (Eq. 7) and

- the gradient information $d f(\phi) / d \phi$ (Eq. 9) from Step 4

Step 6: Set $I=I+1$ and $P=\min \left(P_{\text {end }}, 1+I \Delta P\right)$ where $\Delta P=\left(P_{\text {end }}-\right.$ $\left.P_{\text {start }}\right) / N$

Step 7: If $I<N$ repeat Steps 2 through 7, else continue to Step 8

Step 8: If $f(\phi)$ has converged (Eq. 12) then stop, else repeat Steps 2 through 


\section{Validation and Mesh Independence}

Table 1 shows the mesh refinement study and optimized architectures for various mesh sizes. The topologies can be seen as tree-like structures with primary "V-shape" branches (extending from the isothermal boundary to the upper corners of the domain) and smaller secondary branches extended from the primary branches. These topologies are similar to those obtained in the works by Alexandersen et al. [2], Burger et al. [11], Dirker and Meyer [17], Gersborg-Hansen et al. [19], Marck et al. [26] and Zhang and Liu [36], for isotropic material conductivities.

At a mesh size of $100 \times 100$ the main "V-shape" branches have been established and further mesh refinement yields changes only in the secondary branches. The objective function $\left(T_{\text {ave }}\right)$ is seen to be insensitive (varies by $\sim 1 \%$ ) to further mesh refinement when 120 elements are used in both the $x$ and $y$ directions.

The thermal performance of the system can also be described by a non-dimensional temperature measure, as shown in other investigations [11, 17, 23, 37], given as:

$$
\tau_{\max }=\frac{\left(T_{\max }-T_{0}\right)\left|\mathbf{k}_{\mathbf{0}}\right|}{\dot{q}_{0} L H}
$$

where lower $\tau_{\max }$ values indicate a better thermal performance of the system while higher $\tau_{\max }$ values represent a poorer thermal performance.

Consider Table $2 \mathrm{a}$ where the isothermal boundary temperature $T_{0}$ is varied, while the other parameters $\dot{q}_{0} ; L H$; and $\left|\mathbf{k}_{\mathbf{0}}\right|$ are kept constant, resulting in the same non-dimensional thermal performance of the system and the same optimized architectures. Similarly, in Tables $2 \mathrm{~b}$ to $2 \mathrm{~d}$, the respective parameters $\dot{q}_{0} ; L H$; and $\left|\mathbf{k}_{\mathbf{0}}\right|$ and $\left|\mathbf{k}_{\mathbf{1}}\right|$ (for the same $k_{r}$ ) are varied independently, again resulting in the same non-dimensional thermal performance and the 
same optimized architectures.

Table 2 thus shows the results of validating the in-house FVM code against Eq. 13 by showing that the non-dimensional thermal performance is independent of $T_{0}$ (Table $2 \mathrm{a}$ ); directly proportional to the heat generation rate $\dot{q}$ (Table $2 \mathrm{~b}$ ) and the domain sizes $L$ and $H$ (Table 2c); and inversely proportional to the thermal conductive $\left|\mathbf{k}_{\mathbf{0}}\right|$ (Table $2 \mathrm{~d}$ ).

The in-house FVM code was also validated against OpenFOAM [28, 29], a well established open source FVM package, for various other case studies. For these case studies a maximum error of less than $1 e-6$ was obtained when comparing the results obtained from the in-house code to the results obtained from OpenFOAM.

\section{Numerical Results}

\subsection{Isotropic Conduit Material}

The most practical approach of augmenting the thermal performance is by introducing a high thermal conductive isotropic conduit material $\left(\Omega_{1}\right)$ to an orthotropic heat-generating material $\left(\Omega_{0}\right)$.

Figure 3 shows the non-dimensional thermal performance $\tau_{\max }$ of the system for various heat-generating material angles $\theta_{0}$ at various conductivity ratios $k_{r}$. The non-dimensional thermal performance tends to be improved for heat-generating material angles $10^{\circ} \leq \theta_{0} \leq 45^{\circ}$ and the results tend to indicate that material angles $\theta_{0}<10^{\circ}$ and $\theta_{0}>60^{\circ}$ should be avoided, although some numerical "noise" can be seen.

Table 3 shows the corresponding topologies (optimized architectures) to Fig. 3. The non-dimensional thermal performance $\tau_{\max }$ is also given for each topology. Note that a heat-generating material angle $\theta_{0}=45^{\circ}$ corresponds to an isotropic material; $\theta_{0}<45^{\circ}$ to an orthotropic material with higher 
heat transfer in the $x$ direction; and $\theta_{0}>45^{\circ}$ to an orthotropic material with higher heat transfer in the $y$ direction. It can be seen that the "V-shape" branches (extending from the isothermal boundary into the $\Omega_{0}$ domain) tend to "grow" in the direction of highest thermal resistance of the heat-generating material. It is also worth noting that secondary branches are more prevalent at higher conductivity ratios and the change in topology of the primary "Vshape" branches (with respects to $\theta_{0}$ ) is less drastic at higher conductivity ratios $k_{r}$ (consider for example $\left|\mathbf{k}_{\mathbf{1}}\right|=5000$ and $\theta_{0} \geq 30^{\circ}$ ).

\subsection{Orthotropic Conduit Material}

For completeness of this study the effect of engineering and introducing an orthotropic conduit material $\left(\Omega_{1}\right)$ to an isotropic heat-generating material $\left(\Omega_{0}\right)$ is also considered.

Figure 4 shows the non-dimensional thermal performance $\tau_{\max }$ of the system for various conduit material angles $\theta_{1}$ at various heat-generating material angles $\theta_{0}$. A conduit material angle $\theta_{1} \approx 65^{\circ}$ yields an optimal nondimensional thermal performance with a $15 \%, 10 \%, 6 \%$, and $6 \%$ improvement (for $\theta_{0}=20^{\circ}, 40^{\circ}, 60^{\circ}$, and $80^{\circ}$ respectively) over that of using an isotropic conduit material $\left(\theta_{1}=45^{\circ}\right)$.

Figure 5 shows the non-dimensional thermal performance $\tau_{\max }$ of the system for various conduit material angles $\theta_{1}$ at various conductivity ratios $k_{r}$. As before, a conduit material angle $\theta_{1} \approx 65^{\circ}$ yields an optimal nondimensional thermal performance with a $9 \%, 13 \%, 24 \%$, and $12 \%$ improvement (for the conductivity ratios 100, 200, 300, and 500 respectively) over that of using an isotropic conduit material $\left(\theta_{1}=45^{\circ}\right)$. The augmented nondimensional thermal performance, in the region of $30^{\circ} \leq \theta_{1} \leq 70^{\circ}$, is also less sensitive to $\theta_{1}$ for all conductivity ratios $k_{r}$.

Table 4 shows the corresponding topologies (optimized architectures) to 
Fig. 5. The non-dimensional thermal performance $\tau_{\max }$ is also given for each topology. As before, a conduit material angle $\theta_{1}=45^{\circ}$ corresponds to an isotropic material; $\theta_{1}<45^{\circ}$ to an orthotropic material with higher heat transfer in the $x$ direction; and $\theta_{1}>45^{\circ}$ to an orthotropic material with higher heat transfer in the $y$ direction. It can be seen that the "V-shape" branches tend to "grow" in the direction of lowest thermal resistance of the conduit material.

Figure 6 show the optimal conduit material angle and non-dimensional thermal performance for various domain sizes (aspect ratios $2 H / L$ ). The aspect ratio is taken as $H / 0.5 \mathrm{~L}$ because the position of the isothermal boundary (length $C$ ), in figure 1, resulted in symmetric topologies about the centre. It can be seen that the optimal conduit material angle $\theta_{1}$ corresponds to $\tan ^{-1}(2 H / L)$, in other words the optimal conduit material conductivity $\mathbf{k}_{\mathbf{1}}$ corresponds to a vector pointing from the center of the isothermal boundary to the upper right corner of the domain. The non-dimensional thermal performance for the optimal conduit material angles shows a 19\%, 3\%, 3\%, 9\%, $18 \%, 37 \%$, and $50 \%$ improvement (for $2 H / L=0.32,0.72,1.28,2.0,3.125$, 5.556 , and 12.5 respectively) over that of using an isotropic conduit material $\left(\theta_{1}=45^{\circ}\right)$.

Table 5 shows the corresponding topologies (optimized architectures) to Fig. 6. The optimal conduit material angle $\theta_{1, \mathrm{opt}}$ and non-dimensional thermal performance $\tau_{\max }$ is also given for each topology. It can be seen that the "V-shape" branches tend to "grow" in the direction of the vector pointing from the center of the isothermal boundary to the upper corners of the domain. For high aspect ratios $2 H / L$ a central "trunk" is first formed before the "V-shape" branches, branch off towards the upper corners. 


\section{Conclusions}

For the optimal distribution of an isotropic conduit material within an orthotropic heat-generating material the results indicate that a heat generating material angle $10^{\circ} \leq \theta_{0} \leq 60^{\circ}$ is preferred and a heat-generating material angle $\theta_{0}<10^{\circ}$ and $\theta_{0}>60^{\circ}$ should be avoided. It was also found that the "V-shape" branches of the conduit material tends to "grow" in the direction of highest thermal resistance of the heat-generating material.

For the optimal distribution of an orthotropic conduit material within an isotropic heat-generating material the results show that an optimal conduit material angle exists giving the best thermal performance (lowest $\tau_{\max }$ ). The optimal conduit material angle remains the same for different conductivity ratios and different heat-generating material angles, and the optimal conduit material angle directly corresponds to the domain aspect ratio, that is $\theta_{1, \mathrm{opt}}=\tan ^{-1}(2 H / L)$. The use of an orthotropic conduit material over that of an isotropic conduit material yields a minimum improvement of $3 \%$ and a maximum improvement of $50 \%$ of the thermal performance. A $50 \%$ improvement of the thermal performance effectively translates to either double the allowable heat generation or half the peak operating temperature of the isotropic heat-generating material. It was also found that the "V-shape" branches of the conduit material tends to "grow" in the direction of lowest thermal resistance of the conduit material and the optimal conduit material angle corresponds to a vector pointing from the center of the isothermal boundary to the upper right corner of the domain.

\section{Acknowledgements}

The authors acknowledge with gratitude the support from the University of Pretoria and the South African National Research Foundation (NRF- 
DST).

\section{References}

[1] A. Alebrahim, A. Bejan, Constructal trees of circular fins for conductive and convective heat transfer, International Journal of Heat and Mass Transfer 42 (1999) 3585-3597.

[2] J. Alexandersen, N. Aage, C.S. Andreasen, O. Sigmund, Topology optimisation for natural convection problems, International Journal for Numerical Methods in Fluids 76 (2014) 699-721.

[3] J. Alexandersen, O. Sigmund, N. Aage, Large scale three-dimensional topology optimisation of heat sinks cooled by natural convection, International Journal of Heat and Mass Transfer 100 (2016) 876-891.

[4] M. Almogbel, A. Bejan, Constructal optimization of nonuniformly distributed tree-shaped flow structures for conduction, International Journal of Heat and Mass Transfer 44 (2001) 4185-4194.

[5] R. Barrett, M.W. Berry, T.F. Chan, J. Demmel, J. Donato, J. Dongarra, V. Eijkhout, R. Pozo, C. Romine, H. Van der Vorst, Templates for the solution of linear systems: building blocks for iterative methods, volume 43, Siam, 1994.

[6] S. Behnel, R. Bradshaw, C. Citro, L. Dalcin, D. Seljebotn, K. Smith, Cython: The best of both worlds, Computing in Science Engineering 13 (2011) 31-39.

[7] A. Bejan, Constructal-theory network of conducting paths for cooling a heat generating volume, International Journal of Heat and Mass Transfer 40 (1997) 799-816. 
[8] A. Bejan, M. Almogbel, Constructal t-shaped fins, International Journal of Heat and Mass Transfer 43 (2000) 2101-2115.

[9] M. Bendsoe, O. Sigmund, Topology Optimization: Theory, Methods, and Applications, Springer Berlin Heidelberg, 2003.

[10] R. Boichot, L. Luo, Y. Fan, Tree-network structure generation for heat conduction by cellular automaton, Energy Conversion and Management 50 (2009) 376-386.

[11] F.H. Burger, J. Dirker, J.P. Meyer, Three-dimensional conductive heat transfer topology optimisation in a cubic domain for the volume-tosurface problem, International Journal of Heat and Mass Transfer 67 (2013) 214-224.

[12] X. Cheng, Z. Li, Z. Guo, Constructs of highly effective heat transport paths by bionic optimization, Science in China Series E: Technological Sciences 46 (2003) 296-302.

[13] A. Da Silva, C. Vasile, A. Bejan, Disc cooled with high-conductivity inserts that extend inward from the perimeter, International journal of heat and mass transfer 47 (2004) 4257-4263.

[14] J. Dirker, A.G. Malan, J.P. Meyer, Thermal characterisation of rectangular cooling shapes in solids, International Journal of Numerical Methods for Heat \& Fluid Flow 17 (2007) 361-383.

[15] J. Dirker, J. Meyer, Thermal characterisation of embedded heat spreading layers in rectangular heat-generating electronic modules, International Journal of Heat and Mass Transfer 52 (2009) 1374-1384. 
[16] J. Dirker, J.P. Meyer, Heat removal from power electronics in two direction sets using embedded solid state cooling layers a proposed nonnumerical calculation method, Heat Transfer Engineering 30 (2009) 452465.

[17] J. Dirker, J.P. Meyer, Topology optimization for an internal heatconduction cooling scheme in a square domain for high heat flux applications, Journal of Heat Transfer 135 (2013) 111010.

[18] T. Gao, W. Zhang, J. Zhu, Y. Xu, D. Bassir, Topology optimization of heat conduction problem involving design-dependent heat load effect, Finite Elements in Analysis and Design 44 (2008) 805-813.

[19] A. Gersborg-Hansen, M.P. Bendsøe, O. Sigmund, Topology optimization of heat conduction problems using the finite volume method, Structural and multidisciplinary optimization 31 (2006) 251-259.

[20] S.G. Johnson, Notes on adjoint methods for 18.336 (2012).

[21] E. Jones, T. Oliphant, P. Peterson, et al., SciPy: Open source scientific tools for Python, http://www.scipy.org/, 2001-. [Online; accessed 2015-09-28].

[22] D. Kraft, Algorithm 733: Tomp-fortran modules for optimal control calculations, ACM Transactions on Mathematical Software (TOMS) 20 (1994) 262-281.

[23] G. Ledezma, A. Bejan, Constructal three-dimensional trees for conduction between a volume and one point, Journal of heat transfer 120 (1998) 977-984. 
[24] Q. Li, G.P. Steven, O.M. Querin, Y. Xie, Shape and topology design for heat conduction by evolutionary structural optimization, International Journal of Heat and Mass Transfer 42 (1999) 3361-3371.

[25] Q. Li, G.P. Steven, Y. Xie, O.M. Querin, Evolutionary topology optimization for temperature reduction of heat conducting fields, International Journal of Heat and Mass Transfer 47 (2004) 5071-5083.

[26] G. Marck, M. Nemer, J.L. Harion, S. Russeil, D. Bougeard, Topology optimization using the simp method for multiobjective conductive problems, Numerical Heat Transfer, Part B: Fundamentals 61 (2012) 439470.

[27] M. Neagu, A. Bejan, Constructal placement of high-conductivity inserts in a slab: optimal design of "roughness", Journal of heat transfer 123 (2001) 1184-1189.

[28] OpenCFD Ltd, OpenFOAM: The open source cfd toolbox, http://www . openfoam.com, 2015. [Online; accessed 2015-10-08].

[29] OpenCFD Ltd, OpenFOAM: User guide, http://www.openfoam.com/ docs/user/, 2015. [Online; accessed 2015-10-08].

[30] Python Software Foundation, Python programming language - official website, http://www.python.org/, 1995-. [Online; accessed 2015-09$30]$.

[31] L. Rocha, S. Lorente, A. Bejan, Conduction tree networks with loops for cooling a heat generating volume, International journal of heat and mass transfer 49 (2006) 2626-2635. 
[32] B. Song, Z. Guo, Robustness in the volume-to-point heat conduction optimization problem, International Journal of Heat and Mass Transfer 54 (2011) 4531-4539.

[33] G. Strang, Computational science and engineering, volume 1, WellesleyCambridge Press Wellesley, 2007.

[34] K. Svanberg, The method of moving asymptotes- a new method for structural optimization, International journal for numerical methods in engineering 24 (1987) 359-373.

[35] X. Xu, X. Liang, J. Ren, Optimization of heat conduction using combinatorial optimization algorithms, International journal of heat and mass transfer 50 (2007) 1675-1682.

[36] Y. Zhang, S. Liu, Design of conducting paths based on topology optimization, Heat and Mass Transfer 44 (2008) 1217-1227.

[37] S. Zhou, L. Chen, F. Sun, Optimization of constructal volume-point conduction with variable cross section conducting path, Energy conversion and management 48 (2007) 106-111. 


\section{Figure Captions:}

Fig. 1. 2D computational domain and boundary conditions for the topology optimization problem.

Fig. 2. Material orientation and thermal conductivity.

Fig. 3. Non-dimensional thermal performance for an isotropic conduit material and orthotropic heat-generating material $\left(L=1, H=1 C=0.05, T_{0}=\right.$ $0, \dot{q}_{0}=10, \phi_{\max }=0.1,\left|\mathbf{k}_{\mathbf{0}}\right|=10$ and $\left.\theta_{1}=45^{\circ}\right)$

Fig. 4. Non-dimensional thermal performance for an orthotropic conduit material and orthotropic heat-generating material $(L=1, H=1 C=$ $0.05, T_{0}=0, \dot{q}_{0}=10, \phi_{\max }=0.1,\left|\mathbf{k}_{\mathbf{0}}\right|=10$ and $\left.k_{r}=100\right)$

Fig. 5. Non-dimensional thermal performance for an orthotropic conduit material and isotropic heat-generating material $\left(L=1, H=1 C=0.05, T_{0}=\right.$ $0, \dot{q}_{0}=10, \phi_{\max }=0.1,\left|\mathbf{k}_{\mathbf{0}}\right|=10$ and $\left.\theta_{0}=45^{\circ}\right)$

Fig. 6. Optimal orthotropic conduit material angle and non-dimensional thermal performance for various domain sizes and an isotropic heat-generating material $\left(C=0.05, T_{0}=0, \dot{q}_{0}=10, \phi_{\max }=0.1,\left|\mathbf{k}_{\mathbf{0}}\right|=10, k_{r}=\right.$ 100 , and $\left.\theta_{0}=45^{\circ}\right)$ 


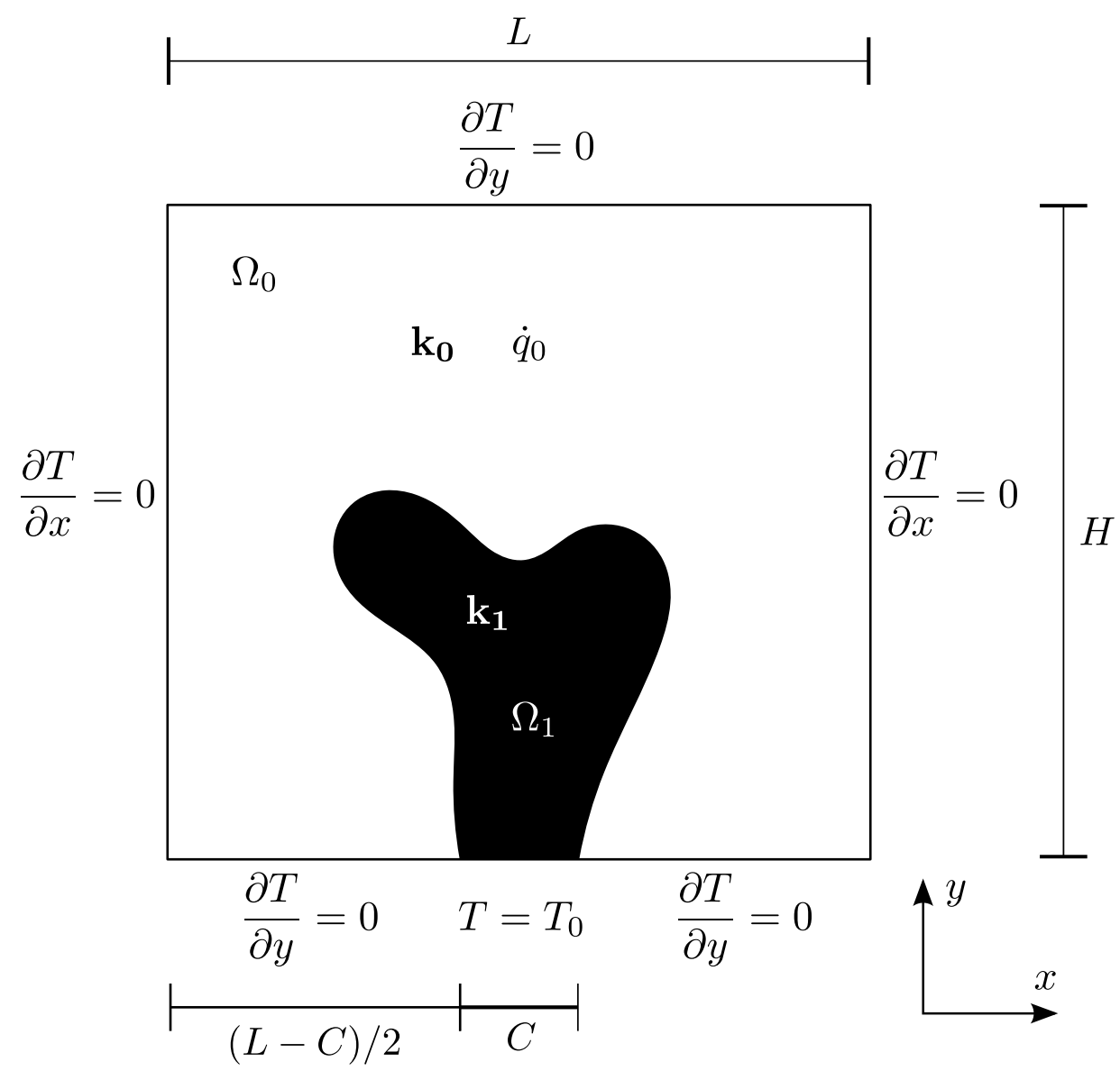

Figure 1: 2D computational domain and boundary conditions for the topology optimization problem. 


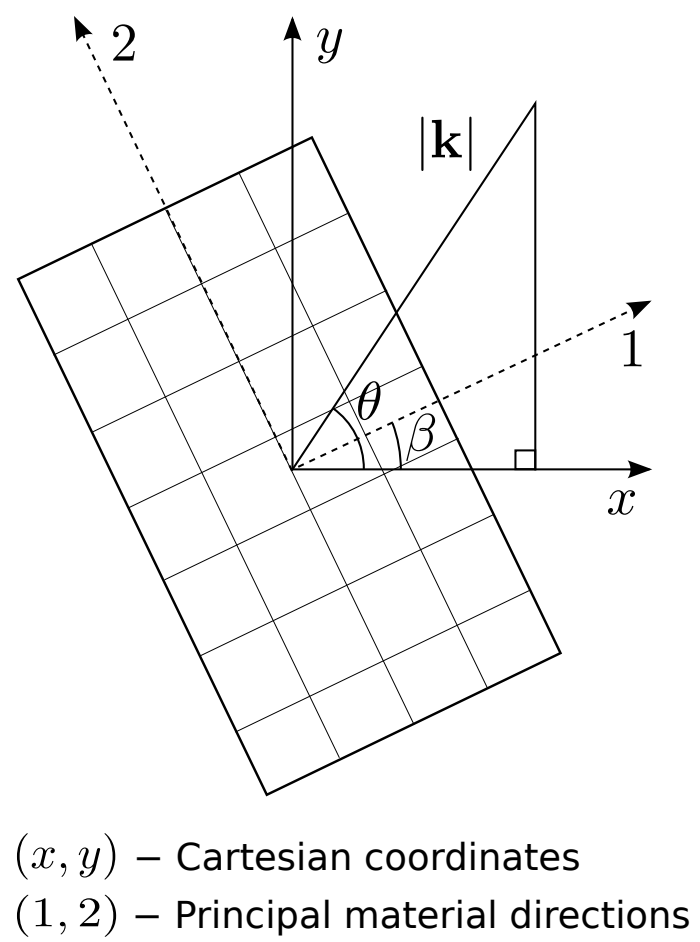

Figure 2: Material orientation and thermal conductivity. 


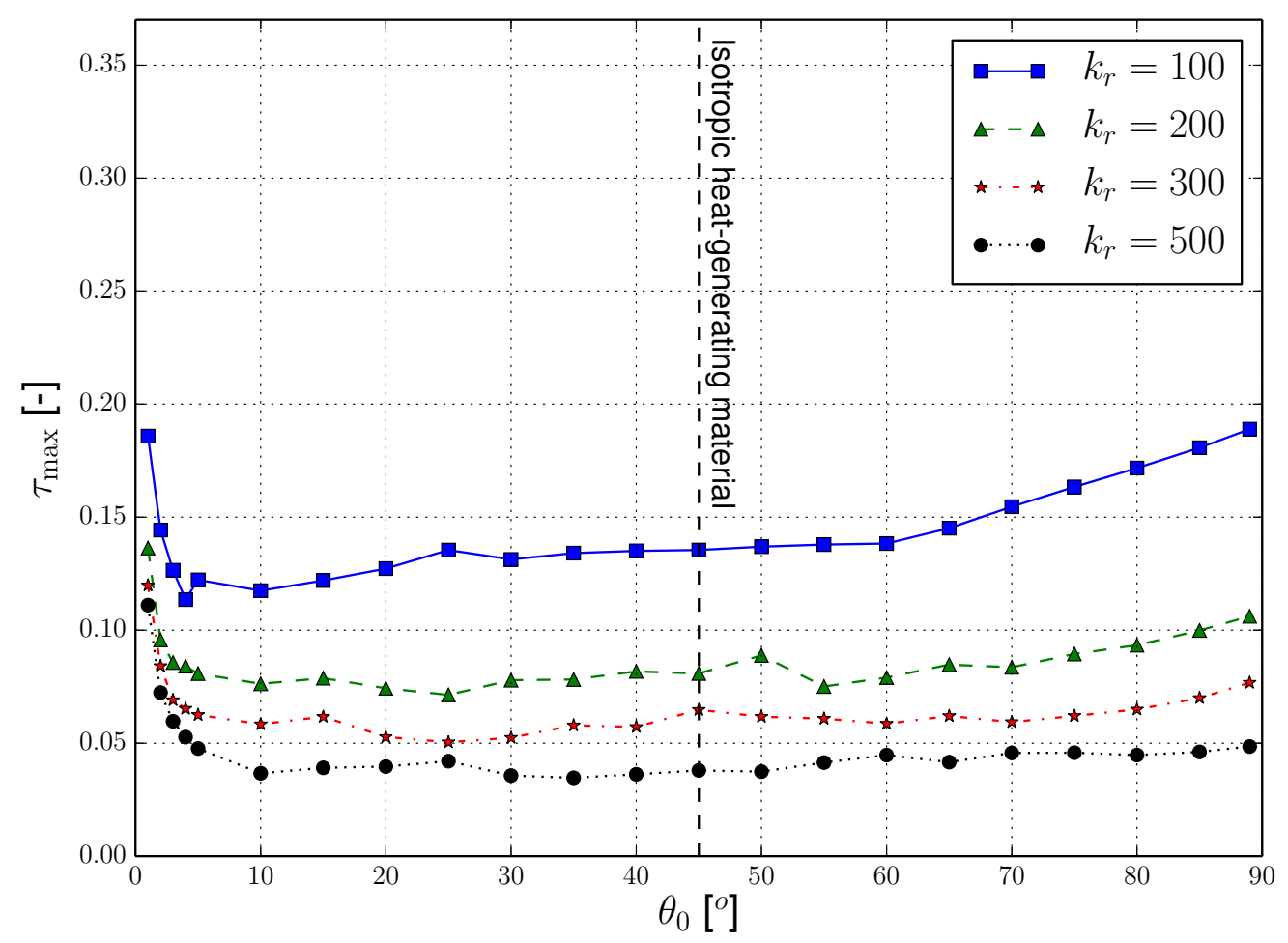

Figure 3: Non-dimensional thermal performance for an isotropic conduit material and orthotropic heat-generating material $\left(L=1, H=1 C=0.05, T_{0}=0, \dot{q}_{0}=10, \phi_{\max }=\right.$ $0.1,\left|\mathbf{k}_{\mathbf{0}}\right|=10$ and $\left.\theta_{1}=45^{\circ}\right)$ 


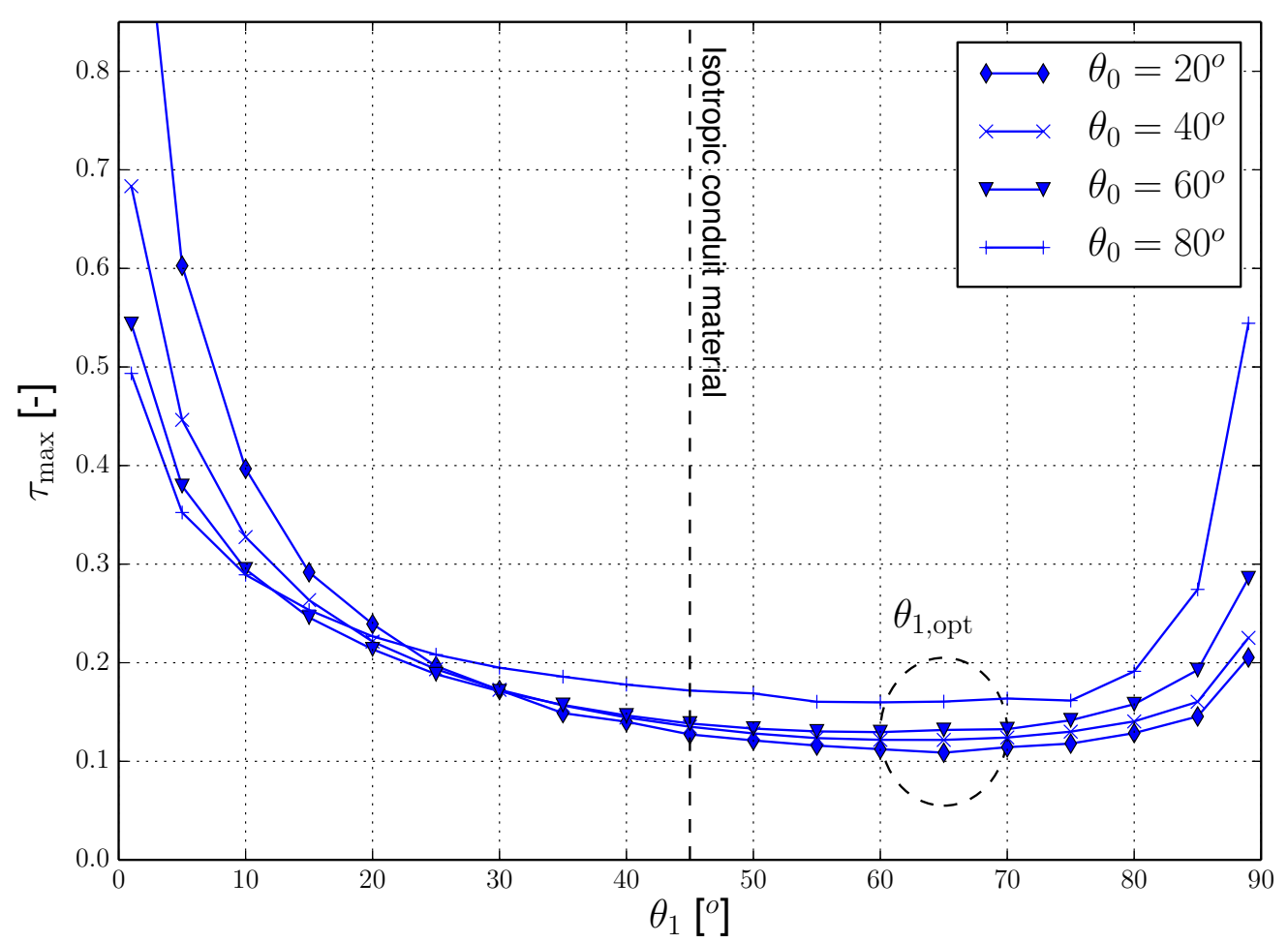

Figure 4: Non-dimensional thermal performance for an orthotropic conduit material and orthotropic heat generating material $\left(L=1, H=1 C=0.05, T_{0}=0, \dot{q}_{0}=10, \phi_{\max }=\right.$ $0.1,\left|\mathbf{k}_{\mathbf{0}}\right|=10$ and $\left.k_{r}=100\right)$ 


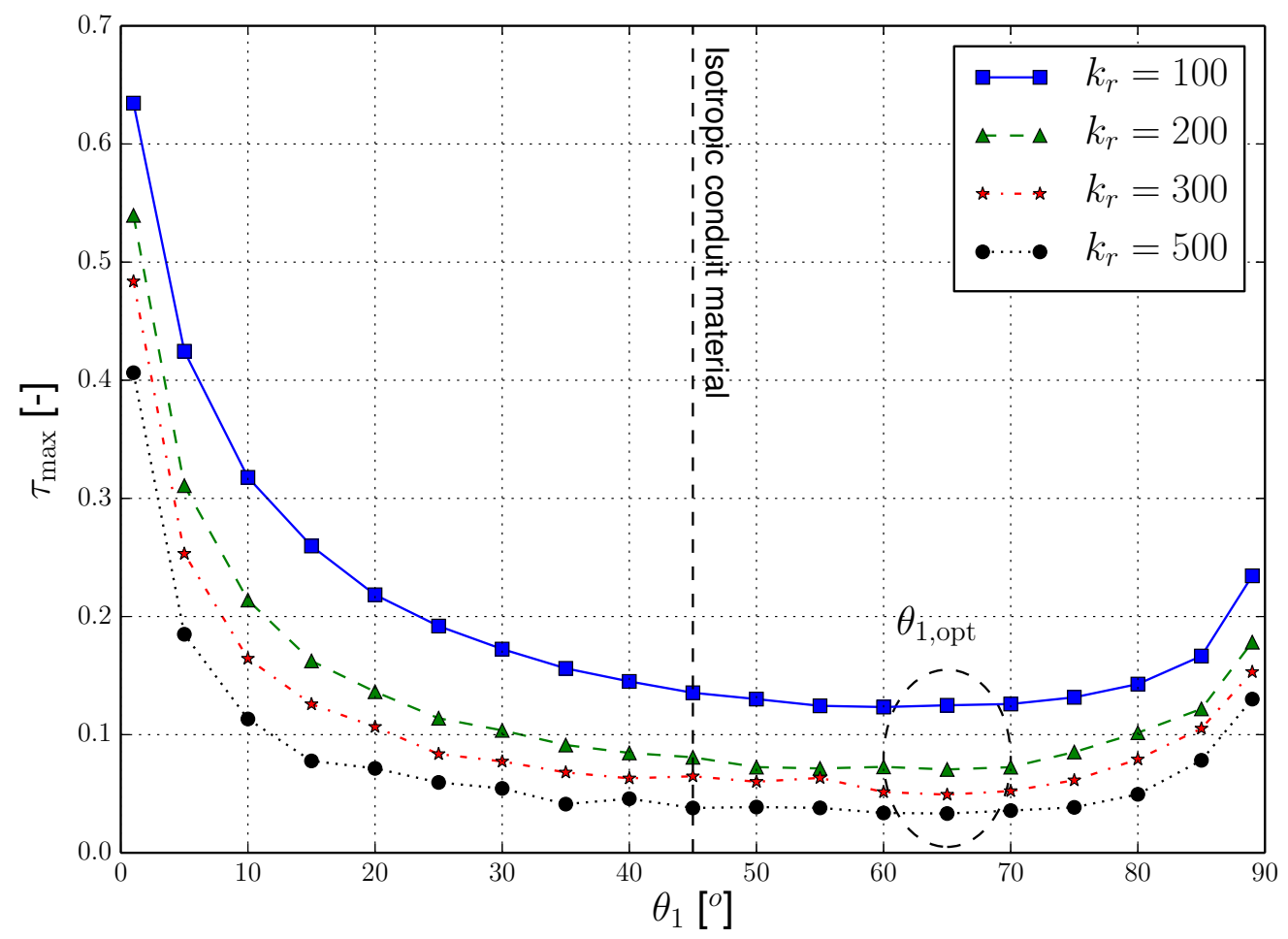

Figure 5: Non-dimensional thermal performance for an orthotropic conduit material and isotropic heat generating material $\left(L=1, H=1 C=0.05, T_{0}=0, \dot{q}_{0}=10, \phi_{\max }=\right.$ $0.1,\left|\mathbf{k}_{\mathbf{0}}\right|=10$ and $\left.\theta_{0}=45^{\circ}\right)$ 


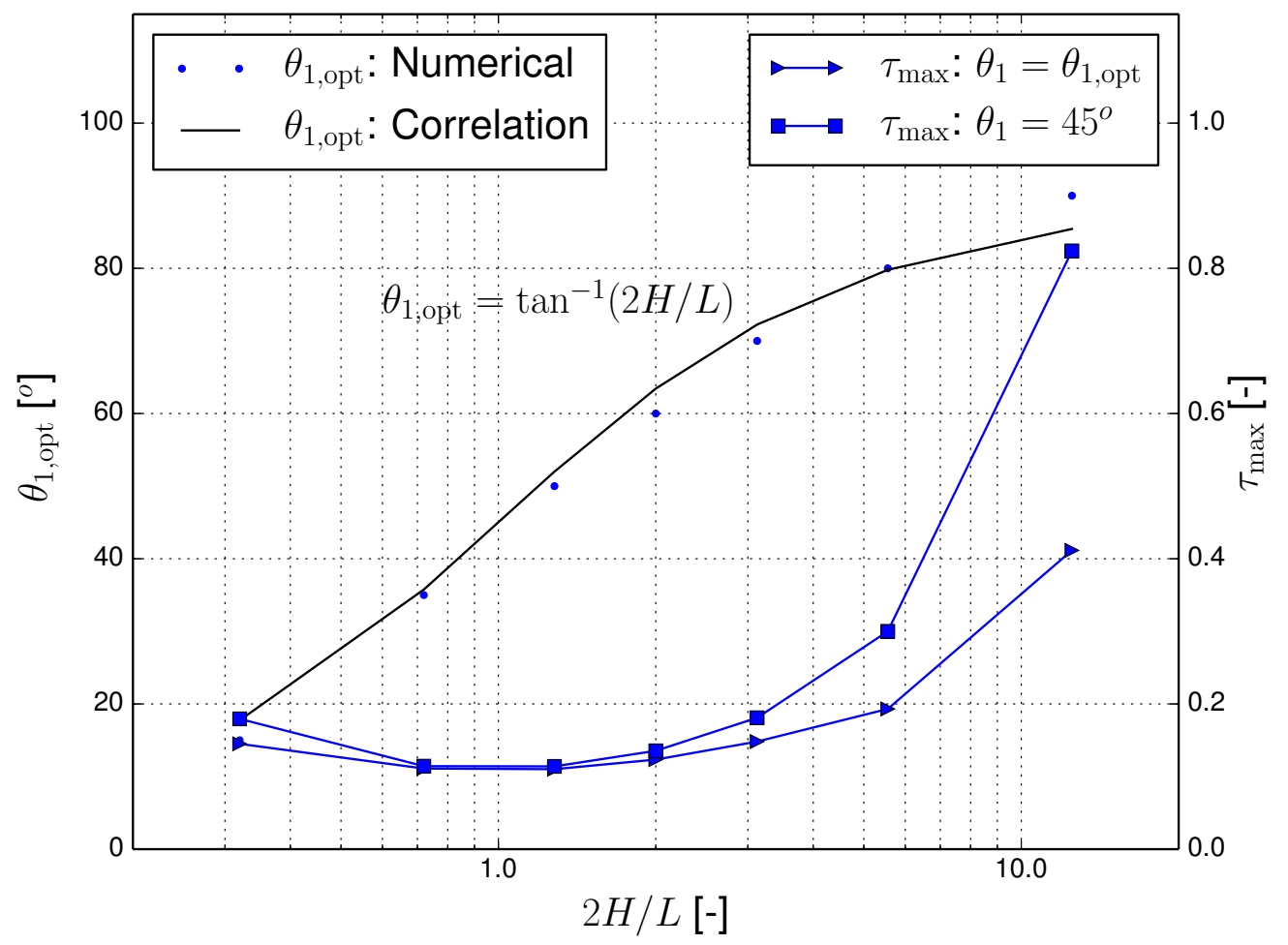

Figure 6: Optimal orthotropic conduit material angle and non-dimensional thermal performance for various domain sizes and an isotropic heat-generating material $\left(C=0.05, T_{0}=\right.$ $0, \dot{q}_{0}=10, \phi_{\max }=0.1,\left|\mathbf{k}_{\mathbf{0}}\right|=10, k_{r}=100$, and $\left.\theta_{0}=45^{\circ}\right)$ 
Table 1: Mesh refinement study $\left(L=1, H=1 C=0.05, T_{0}=0, \dot{q}_{0}=10, \phi_{\max }=\right.$ $0.1,\left|\mathbf{k}_{\mathbf{0}}\right|=10,\left|\mathbf{k}_{\mathbf{1}}\right|=5000, \theta_{0}=20^{\circ}$ and $\left.\theta_{1}=45^{\circ}\right)$

\begin{tabular}{lccccccc}
\hline Size & $20 \times 20$ & $40 \times 40$ & $60 \times 60$ & $80 \times 80$ & $100 \times 100$ & $120 \times 120$ & $140 \times 140$ \\
\hline$T_{\text {ave }}$ & 0.03992 & 0.02666 & 0.02287 & 0.02063 & 0.01878 & 0.01867 & 0.01837 \\
$\frac{T_{\mathrm{ave}}^{i}-T_{\mathrm{ave}}^{i-1}}{T_{\mathrm{ave}}^{i-1}}$ & - & 0.33215 & 0.14241 & 0.09782 & 0.08962 & 0.00609 & 0.01588 \\
\hline Architecture & & &
\end{tabular}


Table 2: Validation study for $L=1, H=1 C=0.05, T_{0}=0, \dot{q}_{0}=10, \phi_{\max }=$ $0.1,\left|\mathbf{k}_{\mathbf{0}}\right|=10,\left|\mathbf{k}_{\mathbf{1}}\right|=5000, \theta_{0}=20^{\circ}$ and $\theta_{1}=45^{\circ}$

(a) Varying $T_{0}$

\begin{tabular}{lrrrrr}
\hline$T_{0}$ & 0 & 5 & 10 & 50 & 100 \\
\hline$\tau_{\max }$ & 0.03965 & 0.03965 & 0.03965 & 0.03965 & 0.03965 \\
\hline
\end{tabular}

(b) Varying $\dot{q}_{0}$

\begin{tabular}{lrrrrr}
\hline$\dot{q}_{0}$ & 1 & 5 & 10 & 50 & 100 \\
\hline$\tau_{\max }$ & 0.03968 & 0.03965 & 0.03965 & 0.03965 & 0.03965 \\
\hline
\end{tabular}

(c) Varying $L$ and $H$

\begin{tabular}{llllll}
\hline$L, H$ & $0.1,0.1$ & $0.5,0.5$ & 1,1 & 5,5 & 10,10 \\
\hline$\tau_{\max }$ & 0.03928 & 0.03965 & 0.03965 & 0.03965 & 0.03965 \\
\hline
\end{tabular}

(d) Varying $\left|\mathbf{k}_{\mathbf{0}}\right|$ and $\left|\mathbf{k}_{\mathbf{1}}\right|$ for $k_{r}=500$

\begin{tabular}{lrrrrr}
\hline$\left|\mathbf{k}_{\mathbf{0}}\right|$ & 0.1 & 1 & 5 & 10 & 50 \\
$\left|\mathbf{k}_{\mathbf{1}}\right|$ & 50 & 500 & 2500 & 5000 & 25000 \\
\hline$\tau_{\max }$ & 0.03965 & 0.03965 & 0.03965 & 0.03965 & 0.03965 \\
\hline
\end{tabular}


Table 3: Topologies for an isotropic conduit material and orthotropic heat-generating material $\left(L=1, H=1 C=0.05, T_{0}=0, \dot{q}_{0}=10, \phi_{\max }=0.1,\left|\mathbf{k}_{\mathbf{0}}\right|=10\right.$ and $\left.\theta_{1}=45^{\circ}\right)$

\begin{tabular}{|c|c|c|c|c|c|c|c|}
\hline$k_{r}=100$ & $\theta_{0}=10$ & $\theta_{0}=20$ & $\theta_{0}=30$ & $\theta_{0}=40$ & $\theta_{0}=50$ & $\theta_{0}=60$ & $\theta_{0}=70$ \\
\hline$\tau_{\max }$ & 0.11748 & 0.12725 & 0.13130 & 0.13509 & 0.13695 & 0.13834 & 0.15464 \\
\hline Archit & & & & & & & \\
\hline
\end{tabular}

\begin{tabular}{llllllll}
\hline \hline$k_{r}=200$ & $\theta_{0}=10$ & $\theta_{0}=20$ & $\theta_{0}=30$ & $\theta_{0}=40$ & $\theta_{0}=50$ & $\theta_{0}=60$ & $\theta_{0}=70$ \\
\hline$\tau_{\max }$ & 0.07626 & 0.07431 & 0.07788 & 0.08176 & 0.08884 & 0.07903 & 0.08360 \\
\hline \multirow{2}{*}{ Architecture } & $\mathbb{V}$
\end{tabular}

\begin{tabular}{|c|c|c|c|c|c|c|c|}
\hline$k_{r}=300$ & $\theta_{0}=10$ & $\theta_{0}=20$ & $\theta_{0}=30$ & $\theta_{0}=40$ & $\theta_{0}=50$ & $\theta_{0}=60$ & $\theta_{0}=70$ \\
\hline$\tau_{\max }$ & 0.05841 & 0.05276 & 0.05241 & 0.05721 & 0.06173 & 0.05864 & 0.05937 \\
\hline Architecture & & & & & & & \\
\hline$k_{r}=500$ & $\theta_{0}=10$ & $\theta_{0}=20$ & $\theta_{0}=30$ & $\theta_{0}=40$ & $\theta_{0}=50$ & $\theta_{0}=60$ & $\theta_{0}=70$ \\
\hline$\tau_{\max }$ & 0.03668 & 0.03965 & 0.03566 & 0.03621 & 0.03741 & 0.04473 & 0.04569 \\
\hline Architecture & & & & & & & \\
\hline
\end{tabular}


Table 4: Topologies for an orthotropic conduit material and isotropic heat-generating material $\left(L=1, H=1 C=0.05, T_{0}=0, \dot{q}_{0}=10, \phi_{\max }=0.1,\left|\mathbf{k}_{\mathbf{0}}\right|=10\right.$ and $\left.\theta_{0}=45^{\circ}\right)$

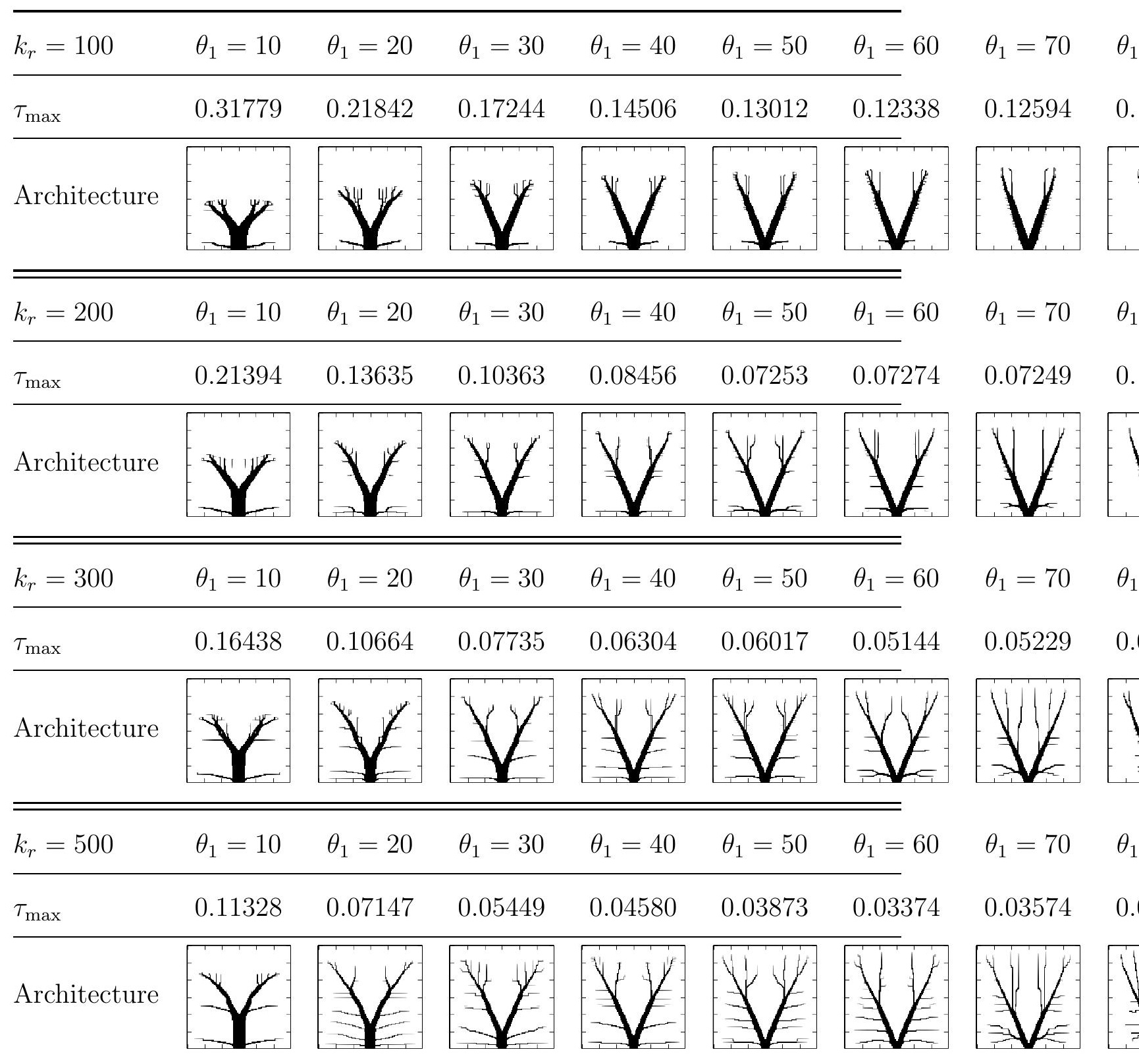


Table 5: Topologies for the optimal orthotropic conduit material angles for various domain sizes and an isotropic heat-generating material $\left(C=0.05, T_{0}=0, \dot{q}_{0}=10, \phi_{\max }=\right.$ $0.1,\left|\mathbf{k}_{\mathbf{0}}\right|=10, k_{r}=100$, and $\left.\theta_{0}=45^{\circ}\right)$

\begin{tabular}{|c|c|c|c|c|c|c|c|}
\hline $2 H / L$ & 0.320 & 0.720 & 1.280 & 2.000 & 3.125 & 5.556 & 12.500 \\
\hline$\theta_{1, \mathrm{opt}}$ & 15 & 35 & 50 & 60 & 70 & 80 & 90 \\
\hline$\tau_{\max }$ & 0.14507 & 0.11104 & 0.11016 & 0.12338 & 0.14822 & 0.19311 & 0.41151 \\
\hline Archit & & & & & & & \\
\hline
\end{tabular}

